\title{
MODULAR FORMS AND QUANTUM INVARIANTS OF 3-MANIFOLDS*
}

\author{
RUTH LAWRENCE ${ }^{\dagger}$ AND DON ZAGIER ${ }^{\ddagger}$
}

1. Introduction. The Witten-Reshetikhin-Turaev (WRT) invariant of a compact connected oriented 3-manifold $M$ may be formally defined by [16]

$$
Z_{k+\check{c}_{\mathfrak{g}}}(M)=\int_{\mathcal{A} / \mathcal{G}} e^{\frac{i k}{4 \pi} \int_{M}\langle A, d A+[A, A] / 3\rangle d \tau} \mathcal{D} A
$$

as a Feynman integral over an infinite dimensional moduli space of $G$-connections on $M$, in terms of additional data, namely a Lie algebra $\mathfrak{g}$ (with dual Coxeter number $\check{c}_{\mathfrak{g}}$ ) and a level $k \in \mathbb{N}$. In this paper we only consider WRT invariants with $\mathfrak{g}=\mathfrak{s l}_{2}$ and it is convenient to put $K=k+2$. This functional integral is not well-defined in a literal sense, being an integral over an infinite dimensional space on which there is no naturally defined measure. However, there are two meaningful statements which can be made about the object represented by the path integral, both of which derive from its form.

1. TQFT: Since the exponent in the integrand (the Chern-Simons action) is additive under gluing of manifolds, one would expect a certain gluing property to be satisfied by the value of $Z_{K}(M)$ with respect to the gluing together of 3-manifolds along a common boundary. Indeed, the invariant $Z_{K}(M)$ fits into a more general picture of invariants of arbitrary links in 3-manifolds (or more generally of slices of these, tangles in 3-manifolds with boundary) and as such describes a functor from the category of Riemann surfaces with punctures, whose morphisms are cobordisms, to the category of vector spaces $([3],[9])$. When this property is formalised, one obtains the mathematical structure of a topological quantum field theory [2].

2. PE: The fact that the dependence on $k$ enters only via a scaling of the exponent in the integrand leads one to expect (as there would be for complex path integrals of a similar form) the existence of a stationary phase expansion for $K$ large of the form $Z_{K}(M) \sim \sum_{A} Z_{K}^{A}(M)$, where the terms $Z_{K}^{A}(M)$ are labelled by the stationary points of the Chern-Simons action (i.e., by the equivalence classes $A$ of flat connections on $M$ ) and each term is the product of an asymptotic series in $K^{-1}$, an exponential in $K$ (determined by the Chern-Simons number for the connection), and a power of $K$ (determined by homological data). There is always one contribution $Z_{K}^{0}(M)$, coming from the trivial connection, which has the form of a formal power series in $K^{-1}$.

For integral values of $K$, however, there is a totally rigorous and combinatorial definition [13] of the invariant using the representation theory of the quantum group $U_{\xi} \mathfrak{s l}_{2}$ at the root of unity $\xi=e^{2 \pi i / K}$. According to [11], $Z_{K}(M) \in \mathbb{Q}[\xi]$ for all rational homology spheres $M$. Moreover, one can construct a kind of perturbative expansion, for $\xi$ tending to 1 through roots of unity of increasing order, by using the congruence properties of the coefficients of the polynomials representing $Z_{K}(M)$ for prime $K$ (see [12]). What is obtained is a formal power series invariant $Z_{\infty}(M) \in \mathbb{Q}[[h]]$ in $h=\xi-1$.

* Received March 31, 1999; accepted for publication April 2, 1999.

† Institute of Mathematics, Hebrew University, 91904 Jerusalem, Israel and Department of Mathematics, University of Michigan, Ann Arbor, MI 48109-1109, USA (ruthel@math.huji.ac.il).

$\ddagger$ Max-Planck-Institut für Mathematik, D-53111 Bonn, Germany (zagier@mpim-bonn.mpg.de). 
This series has been shown [14] to converge $K$-adically to $Z_{K}(M)$ for prime $K$, for all rational homology spheres. Moreover, in some cases it has been found by explicit computations that the power series $Z_{\infty}(M)$ in $K^{-1}$ also describes the asymptotic expansion in the complex sense of the "trivial connection contribution" $Z_{K}^{0}(M)$ as $K \rightarrow \infty$. ([8], [10])

Although the combinatorial definition of [13] makes sense for any root of unity, the definition of [16] has been traditionally only considered for integer (rather than rational) $K$, and in the limit $\xi \rightarrow 1$. In principle one can carry out a radial perturbative expansion near any root of unity, i.e. in a power series in $t$ for $q=\xi e^{-t}$ where $\xi=e^{2 \pi i \alpha}$ with $\alpha \in \mathbb{Q}$. To our knowledge this has yet to be done directly from Witten's integral formulation, although it has been done in special cases in [8] and [10] from explicit formulae for $Z_{K}^{0}(M)$.

The phenomenon which we see here of functions which make sense only at, or infinitesimally near to, roots of unity also occurs in [17] and in connection with "period functions" of modular forms and mock theta functions. The purpose of this paper is to show that in fact these two coincide, at least in certain cases. In particular we will show that, in certain cases, there is a modular form of half-integral weight whose Eichler integral is almost modular, its limiting values near rational points being equal to the WRT-invariants of suitable 3 -manifolds. The obstruction to modularity is measured by a certain cocycle which is an analogue for half-integral weights of the period polynomial for integral weights. See $\S 4$.

For most of the paper we will concentrate on one single example, the Poincare homology sphere $\Sigma(2,3,5)$. The WRT-invariant of this manifold has been calculated in the literature in several different ways and is a particularly beautiful numbertheoretical function. In $\S 2$ we collect a number of these formulae and give a table of values of $W(\xi)$ (a normalised version of $Z_{K}(M)$ ) for roots of unity $\xi$ of small order. In $\S 3$ we obtain a formula of a different sort for $W(\xi)$ by introducing a certain theta series of weight $3 / 2$ and showing that the limiting values of the associated Eichler integral coincide with the numbers $W(\xi)$. This property is used in $\S 4$ to determine the behaviour of the function $W\left(e^{2 \pi i \alpha}\right)(\alpha \in \mathbb{Q})$ under the action of modular transformations. In $\S 5$ we describe briefly a surprising connection, found by Sander Zwegers, between the WRT-invariant of $\Sigma(2,3,5)$ and one of Ramanujan's "mock theta functions of order 5". This leads to yet another formula for $W(\xi)$ as an infinite sum of polynomials in $\xi^{-1}$ which becomes finite (and hence makes sense) exactly when $\xi$ is a root of unity; this is similar to the phenomenon appearing in the "strange identity" of [17]. Finally, in $\S 6$ we describe the generalisation to other Seifert fibrations. If these have exactly three exceptional fibres, then the picture is quite analogous to the one for the Poincaré homology sphere, while in the case of four or more exceptional fibres the same type of ideas apply but the picture becomes more complicated.

Acknowledgements. The authors would like to thank J. Bernstein for suggesting that the formulas which they had separately found in connection with WRT-invariants of simple manifolds and Eichler integrals of modular forms, respectively, might in fact be related. The first author is an Alfred P. Sloan Research Fellow; she would also like to thank the IHES for their hospitality while the main part of this work was done. This work was partially supported by NSF grant DMS-9626544.

2. The WRT-invariant of the Poincaré homology sphere: a collection of formulae. Computation of $Z_{K}(M)$ or $Z_{\infty}(M)$ is generally a hard problem. For $K$ small, there are relations between $Z_{K}(M)$ and classical topological invariants of $M$. For a general 3-manifold it is hopeless to give nice formulae for WRT-invariants, 
although for special classes of manifolds this can and has been done: lens spaces [6], surgery around $(2, n)$-torus knots [7], and Seifert fibred manifolds [10]. The Poincaré homology sphere fits into all but the first of these categories, as well as having a nice realisation as the quotient of $S^{3}$ by the icosahedral group, and hence is particularly accessible to computations.

The WRT-invariant associated to the Poincaré homology sphere $M=\Sigma(2,3,5)$ gives an element $W(\xi)=W_{M}(\xi) \in \mathbb{Z}[\xi]$ for every root of unity $\xi$. This invariant transforms equivariantly under the Galois group, i.e. $W\left(\xi^{\sigma}\right)=W(\xi)^{\sigma}$ for every $\sigma \in$ $\operatorname{Gal}(\mathbb{Q}(\xi) / \mathbb{Q})$, so it is enough to compute $W\left(\zeta_{K}\right)$ for the standard $K$ th root of unity $\zeta_{K}=e^{2 \pi i / K}$. However we will try to write down formulae which work for all roots of unity, since we will later be interested in the behaviour of the function $\mathbb{Q} \rightarrow \mathbb{C}$ defined by $\alpha \mapsto W\left(e^{2 \pi i \alpha}\right)$.

A number of formulae for $W(\xi)$ have been given. We quote in particular several of the formulae from [7], [8], and [10]. In fact we will use a slightly different normalisation from the standardly defined $s l_{2}$ WRT-invariant of compact connected orientable 3manifolds $Z_{K}(M)$ (for which $Z_{K}\left(S^{3}\right)=1$ ) in order to simplify the formulae. In particular, we set

$$
W\left(\zeta_{K}\right)=\zeta_{K}\left(\zeta_{K}-1\right) Z_{K}(\Sigma(2,3,5))
$$

The geometric meaning of the factor $\zeta_{K}\left(\zeta_{K}-1\right)$ is as yet unclear.

First, the manifold $M$ can be obtained from $S^{3}$ by integer surgery around a $(2,-3)$ torus knot with 2 additional twists. Specialising to this case the formula given in [7] for the WRT-invariants of such manifolds and assuming $K$ odd, we obtain the formula

$$
W(\xi)=\frac{1}{G_{-}} \sum_{\substack{m \in \mathbb{Z} / 2 K \mathbb{Z} \\ n \in\left(\mathbb{Z}+\frac{1}{2}\right) / K \mathbb{Z}}}(-1)^{n-\frac{1}{2}} \varepsilon\left(\frac{m}{K}, \frac{n}{K}\right) A^{5 m^{2}+2 m-6 n^{2}-4 n-\frac{1}{2}}
$$

where $A$ is a primitive $4 K$-th root of unity with $A^{4}=\xi, G_{-}$is the Gauss sum $\sum_{1}(-1)^{j} A^{-j^{2}}$, and $\varepsilon:(\mathbb{R} / 2 \mathbb{Z})^{2} \rightarrow\{-1,0,1\}$ is the function defined by $\varepsilon(x, y)=$ $\frac{1}{2}\left((-1)^{[x+y]}+(-1)^{[x-y]}\right)$. (The formula is given in a slightly different form in [7]; to pass from (II.10) there to (1), make the substitutions $a+1= \pm m, j+\frac{1}{2}= \pm n$ and use the symmetry properties.) It is not hard to check from this formula that $W(\xi)$ belongs to $\mathbb{Q}(\xi)$ rather than just $\mathbb{Q}(A)$, and from the identity $G_{-}^{2}=-2 K(-A)^{K}$ it follows that $W(\xi) \in \frac{1}{2 K} \mathbb{Z}[\xi]$, but the integrality of $W(\xi)$ is not obvious from (1). It is known from [11] that for all rational homology spheres, $Z_{K}(M) \in \mathbb{Z}[\xi]$ for prime $K$, so that $W(\xi) \in(\xi-1) \mathbb{Z}[\xi)]$. (See [7] for an explicit derivation for $K$ prime in this case, or [8] for an extention of this to an arbitrary prime power $K$.)

Next, $M$ can also be thought of as a Seifert-fibred manifold, with three exceptional fibres and branching numbers $(2,3,5)$. The formulae proved in [10] specialise in this case to

$$
W(\xi)=\frac{1}{2 G} \sum_{\substack{\beta(\bmod 60 K) \\ \beta \neq 0(\bmod K)}} \frac{\left(1-\zeta^{24 \beta}\right)\left(1-\zeta^{40 \beta}\right)}{1+\zeta^{60 \beta}} \zeta^{-(\beta+1)^{2}},
$$

where $\zeta$ denotes a primitive $120 K$-th root of unity with $\zeta^{120}=\xi$ and $G$ the Gauss sum $\sum_{\beta} \zeta^{-\beta^{2}}$. (The number of terms can be reduced by a factor of 8 by summing only over $\beta$ lying between 0 and $15 K$ and congruent to $K+1$ modulo 2 .) If $\xi=e^{2 \pi i / K}$ 
and $\zeta=e^{\pi i / 60 K}$, then $G=(1-i) \sqrt{30 K}$ and (2) can be rewritten more explicitly as

$$
W\left(\zeta_{K}\right)=-\frac{1+i}{\sqrt{120 K}} \sum_{\substack{\beta(\bmod 60 K) \\ \beta \neq 0(\bmod K)}} \frac{\sin (\pi \beta / 3 K) \sin (\pi \beta / 5 K)}{\cos (\pi \beta / 2 K)} e^{-\pi i\left(\beta^{2}+1\right) / 60 K},
$$

Finally, there is an even simpler formula for $W(\xi)$ which will follow from the results in $\S 3$ :

$$
W(\xi)=1-\frac{1}{2} \sum_{n=1}^{30 K} \chi_{+}(n)\left(1-\frac{n}{30 K}\right) \xi^{\left(n^{2}-1\right) / 120},
$$

where $\chi_{+}(n)$ is the periodic function defined by the table

$$
\begin{array}{l|rrrrrrrrc}
n(\bmod 60) & 1 & 11 & 19 & 29 & 31 & 41 & 49 & 59 & \text { (other) } \\
\hline \chi_{+}(n) & 1 & 1 & 1 & 1 & -1 & -1 & -1 & -1 & 0
\end{array}
$$

Any of the formulae (1)-(4) can be used to compute $W(\xi)$. By virtue of Galois invariance, it suffices to give $W(\xi)$ as a polynomial in $\xi$ for each value of $K$, where $\xi$ is a primitive $K$ th root of unity. Here is a table up to $K=12$.

\begin{tabular}{c|c}
$K$ & $W(\xi)$ \\
\hline 1 & 0 \\
2 & 2 \\
3 & $-2 \xi-1$ \\
4 & $\xi+1$ \\
5 & $-\xi^{3}-2 \xi^{2}-2 \xi$ \\
6 & -1
\end{tabular}

\begin{tabular}{c|c}
$K$ & $W(\xi)$ \\
\hline 7 & $-\xi^{5}-\xi^{4}-3 \xi^{3}-\xi^{2}-1$ \\
8 & $\xi^{3}-2 \xi^{2}-2 \xi+1$ \\
9 & $\xi^{4}-\xi^{3}+\xi^{2}+\xi+1$ \\
10 & $-3 \xi^{3}-2 \xi+2$ \\
11 & $-\xi^{9}+\xi^{8}+\xi^{3}-\xi$ \\
12 & $-3 \xi^{2}+\xi+4$
\end{tabular}

As mentioned in the Introduction, there is a second version of the function $W(q)$ which, instead of being a function $\xi \mapsto W(\xi)$ defined. at roots of unity, is a formal power series in $q-1$ with rational coefficients. There are two ways of obtaining this power series, one using $p$-adic ideas and the other purely complex. We describe this briefly. A result of Ohtsuki [12] implies (for arbitrary rational homology spheres $M$ ) that there exist rational numbers $\lambda_{n}$ such that for all sufficiently large prime $K$,

$$
\lambda_{n} \equiv\left[W\left(\zeta_{K}\right)\right]_{h^{n}} \quad \bmod K
$$

where the square brackets denote the coefficient of $h^{n}$ in the integer polynomial in $h=\zeta_{K}-1$ representing $W\left(\zeta_{K}\right)$. Define a formal power series,

$$
W_{\infty}(h):=\sum_{n=0}^{\infty} \lambda_{n} h^{n} \in \mathbb{Q}[[h]] .
$$

According to [7] and [8], for the Poincaré homology sphere,

$$
2(1+h)^{\frac{1}{120}} W_{\infty}(h)=(1+h)^{30\left(\bar{B}-\frac{4}{15}\right)^{2}}-(1+h)^{30\left(\bar{B}-\frac{1}{15}\right)^{2}},
$$

and $W_{\infty}(h)$ converges $p$-adically to $W\left(\zeta_{K}-1\right)$ for all $K$ which are powers of a prime number $p$. (This makes sense because the $p$-adic valuation of the algebraic number 
$\zeta_{K}-1$ is then strictly positive.) The right hand side of (6) is to be understood according to the binomial expansion, with the powers of the symbol $\bar{B}$ being formally replaced by modified Euler numbers whose generating function is $e^{z \bar{B}}=\operatorname{sech} \frac{z}{4}$. For the Poincaré sphere this power series begins

$$
\begin{aligned}
W_{\infty}(h)=h & -5 h^{2}+39 h^{3}-419 h^{4}+5760 h^{5}-96592 h^{6}+1912421 h^{7} \\
& -43664112 h^{8}+1129444381 h^{9}-32643929747 h^{10}+\cdots
\end{aligned}
$$

(The corresponding series for the original normalisation $Z_{\infty}(h)=W_{\infty}(h) / h(1+h)$ of the WRT-invariant, as given in [7], begins $1-6 h+45 h^{2}-464 h^{3}+6224 h^{4}-$ $102816 h^{5}+\cdots$.) Note that this power series has integral coefficients; the corresponding fact is known to be true for all integer homology spheres, but no general topological interpretation of these coefficients is known.

Finally, according to [7] and [10], the power series $W_{\infty}$ may also be expressed as the asymptotic expansion near $q=1$ of the integrals:

$$
\begin{aligned}
q^{1 / 120} W_{\infty}(q-1) & \sim \int_{-\infty}^{\infty} \frac{q^{-2(15 i z-4)^{2} / 15}-q^{-2(15 i z-1)^{2} / 15}}{\cosh 2 \pi z} d z \\
& \sim \frac{1+i}{2 \sqrt{120 K}} \int_{C} \frac{\left(q^{\beta / 6}-q^{-\beta / 6}\right)\left(q^{\beta / 10}-q^{-\beta / 10}\right)}{q^{\beta / 4}+q^{-\beta / 4}} q^{-\beta^{2} / 120} d \beta,
\end{aligned}
$$

where the contour $C$ in the second integral passes through the origin in the direction of steepest descent $\sqrt{-i K}$. In fact, in the case of the Poincaré homology sphere, there are precisely three terms in the stationary phase decomposition, the term $W^{0}(\xi)$ associated with the trivial connection being given by the last integral in (7) (with $q=\xi$, and multiplied by $\xi^{-1 / 120}$ ) and the terms corresponding to the two non-trivial flat connections being given [4] by

$$
W^{j}\left(\zeta_{K}\right)=2 \sqrt{\frac{K}{5 i}} e^{-\pi i / 60 K} \sin \left(\frac{\pi j}{5}\right) e^{-\pi i m_{j}^{2} K / 60} \quad(j=1,2),
$$

where $m_{1}^{2}=1, m_{2}^{2}=49$ come from the corresponding values of the Chern-Simons action. In this case, and indeed for all Seifert fibred manifolds [10], the decomposition $Z_{K}(M)=\sum_{A} Z_{K}^{A}(M)$ holds not only perturbatively amongst formal power series, but as an exact expression at all integers $K$.

From the integral formulae (7), or from the computations given later in this paper, one finds the following alternative explicit expression for the power series $W_{\infty}(h)$ :

$$
\begin{aligned}
1-W_{\infty}\left(e^{-t}-1\right) & =e^{t / 120} \sum_{r=0}^{\infty} \frac{a_{r}}{r !}\left(\frac{t}{120}\right)^{r} \\
= & 1+t+\frac{9}{2} t^{2}+\frac{205}{6} t^{3}+\frac{2907}{8} t^{4}+\frac{596341}{120} t^{5}+\cdots,
\end{aligned}
$$

where the coefficients $a_{r} \in \mathbb{Z}$ are defined by

$$
\begin{aligned}
& \sum_{r=0}^{\infty} \frac{a_{r}}{(2 r) !} x^{2 r}=\frac{\cos (5 x) \cos (9 x)}{\cos (15 x)} \\
& \quad=1+\frac{119}{2} x^{2}+\frac{129361}{24} x^{4}+\frac{353851559}{720} x^{6}+\frac{258138625303}{5760} x^{8}+\cdots
\end{aligned}
$$


3. The invariant $W(\xi)$ as the limit of a holomorphic function.

$$
A(q)=\sum_{n=1}^{\infty} \chi_{+}(n) q^{\left(n^{2}-1\right) / 120}=1+q+q^{3}+q^{7}-q^{8}-q^{14}-q^{20}-\cdots \quad(|q|<1),
$$

a holomorphic function in the unit disk.

THEOREM 1. Let $\xi$ be a root of unity. Then the radial limit of $1-\frac{1}{2} A(q)$ as $q$ tends to $\xi$ equals $W(\xi)$, the (rescaled) WRT-invariant of the Poincaré homology sphere.

THEOREM 2. The asymptotic expansion of $1-\frac{1}{2} A(q)$ as $q \nearrow 1$ is the power series $W_{\infty}(q-1)$.

The proofs of both these results make use of the following general proposition.

Proposition. Let $C: \mathbb{Z} \rightarrow \mathbb{C}$ be a periodic function with mean value 0 . Then the associated L-series $L(s, C)=\sum_{n=1}^{\infty} C(n) n^{-s}(\Re(s)>1)$ extends holomorphically to all of $\mathbb{C}$ and the two functions $\sum_{n=1}^{\infty} C(n) e^{-n t}$ and $\sum_{n=1}^{\infty} C(n) e^{-n^{2} t}(t>0)$ have the asymptotic expansions

$$
\sum_{n=1}^{\infty} C(n) e^{-n t} \sim \sum_{r=0}^{\infty} L(-r, C) \frac{(-t)^{r}}{r !}, \quad \sum_{n=1}^{\infty} C(n) e^{-n^{2} t} \sim \sum_{r=0}^{\infty} L(-2 r, C) \frac{(-t)^{r}}{r !}
$$

as $t \searrow 0$. The numbers $L(-r, C)$ are given explicitly by

$$
L(-r, C)=-\frac{M^{r}}{r+1} \sum_{n=1}^{M} C(n) B_{r+1}\left(\frac{n}{M}\right) \quad .(r=0,1, \ldots)
$$

where $B_{k}(x)$ denotes the $k$-th Bernoulli polynomial and $M$ is any period of the function $C(n)$.

Corollary. Let $C(n)$ and $M$ be as in the Proposition. Then each of the expressions

$$
\left.\sum_{n=1}^{\infty} C(n) e^{-n t}\right|_{t=0},\left.\quad \sum_{n=1}^{\infty} C(n) e^{-n^{2} t}\right|_{t=0},\left.\quad \sum_{n=1}^{\infty} C(n) n^{-s}\right|_{s=0}
$$

(where the first two denote limits as $t \searrow 0$ and the third the analytic continuation to $s=0)$ define the same "renormalised sum" $\sum_{n \geq 1}^{*} C(n)$ and the value of this sum is given by

$$
\sum_{n \geq 1}^{*} C(n)=-\sum_{n=1}^{M}\left(\frac{n}{M}-\frac{1}{2}\right) C(n)
$$

Remark. The expansion of $\sum C(n) e^{-n^{2} t}$ involves only the values of $L(s, \chi)$ at negative even integers. If $C$ is an odd periodic function, the values at negative odd integers vanish anyway, since $\sum C(n) e^{-n t}=\sum_{0<n<M / 2} C(n) \sinh ((M / 2-n) t) / \sinh (M t / 2)$, which has an even power series expansion at the origin. If $C$ is even, then the same argument shows that $L(-2 r, C)=0$ for all $r>0$ and hence that the asymptotic expansion of $\frac{1}{2} C(0)+\sum_{n \geq 1} C(n) e^{-n^{2} t}$ at the origin is identically 0 , but this is obvious anyway since this sum is one-half the sum over all $n \in \mathbb{Z}$ and hence is $\mathrm{O}\left(e^{-A / t}\right)$ for some $A>0$ by the Poisson summation formula. 
Proof of the Proposition. We give only an indication, since the Proposition is more or less standard. By summing the geometric series we see that

$$
\sum_{n=1}^{\infty} C(n) e^{-n t}=\sum_{n=1}^{M} C(n) \frac{e^{-n t}}{1-e^{-M t}}=-\sum_{n=1}^{M} C(n) \sum_{r=-1}^{\infty} \frac{B_{r+1}(n / M)}{(r+1) !}(-M t)^{r}
$$

$$
(|t|<2 \pi / M)
$$

and hence that $\sum_{n \geq 1} C(n) e^{-n t}$ has an asymptotic expansion of the form $\sum_{r>0} b_{r}(-t)^{r} / r$ ! with $\bar{b}_{r}$ equal to the expression on the right-hand side of (12). On the other hand, from the Mellin integral representation

$$
\Gamma(s) L(s, C)=\int_{0}^{\infty}\left(\sum_{n=1}^{\infty} C(n) e^{-n t}\right) t^{s-1} d t \quad(\Re(s)>1)
$$

we find (for any $A \in \mathbb{R}_{>0}$ and $R \in \mathbb{Z}_{\geq 0}$ )

$$
\begin{aligned}
\Gamma(s) L(s, C) & =\int_{0}^{A}\left(\sum_{r=0}^{R-1} \frac{b_{r}}{r !}(-t)^{r}+\mathrm{O}\left(t^{R}\right)\right) t^{s-1} d t+\int_{A}^{\infty} \mathrm{O}\left(e^{-t}\right) t^{s-1} d t \\
& \left.=\sum_{r=0}^{R-1} \frac{(-1)^{r} b_{r}}{r !} \frac{1}{s+r}+\text { (holomorphic in } \Re(s)>-R\right),
\end{aligned}
$$

and this gives the analytic continuation of $L(s, C)$ and (by comparison of residues on both sides) the identity $L(-r, C)=b_{r}(r \geq 0)$. Finally, the same argument applied to the Mellin transform integral

$$
\int_{0}^{\infty}\left(\sum_{n=1}^{\infty} C(n) e^{-n^{2} t}\right) t^{s-1} d t=\Gamma(s) L(2 s, C) \quad\left(\Re(s)>\frac{1}{2}\right)
$$

shows that, if the function $\sum_{n \geq 1} C(n) e^{-n^{2} t}$ has an asymptotic expansion of the form $\sum_{r>0} c_{r}(-t)^{r} / r$ ! as $t \searrow 0$, then $c_{r}$ is indeed equal to $L(-2 r, C)$ as asserted in the Proposition. We can show that such an asymptotic expansion exists in several ways, e.g. by applying a "shifted version" of the Euler-Maclaurin summation formula to the function $f(x)=e^{-t x^{2}}$ or by writing $\sum C(n) e^{-n^{2} t}$ as a contour integral $\frac{1}{2 \pi i} \int_{\gamma} \Gamma(s) L(2 s, C) t^{-s} d t$ for a contour $\gamma$ encircling the negative imaginary axis and then moving this contour to the left across the simple poles at $s=0,-1, \ldots$

Proof of Theorem 1. We apply the Proposition to $C(n)=\chi_{+}(n) \xi^{\left(n^{2}-1\right) / 120}$, where $\chi_{+}(n)$ is the function defined by (5) and $\xi$ any root of unity. Observe that if $K$ is the order of $\xi$ then the number $M=60 K$ is a period for $C(n)$ while $C(60 K-n)=-C(n)$ so that the mean value of $C(n)$ is zero. Denote the corresponding Dirichlet series by $\widetilde{L}_{\xi}\left(s, \chi_{+}\right)$. The proposition and its corollary immediately give that the function $e^{-t / 120} A\left(\xi e^{-t}\right)$ has an asymptotic expansion $\sum_{r \geq 0} \widetilde{L}_{\xi}\left(-2 r, \chi_{+}\right)(-t / 120)^{r} / r$ ! at $t=0$ and that the limiting value

$$
A(\xi):=\lim _{t \searrow_{0}} A\left(\xi e^{-t}\right)=\widetilde{L}_{\xi}\left(0, \chi_{+}\right)
$$

is given by the sum in (4). 
To complete the proof of the theorem, we must relate the value in (4) to the sum in (2). Replacing $\beta$ by $\beta-n$ in the sum defining the Gauss sum $G$ in (2), we find

$$
G=\sum_{\beta \bmod 60 K} \zeta^{-(\beta-n)^{2}}=\zeta^{-n^{2}} \sum_{\beta \bmod 60 K} \zeta^{-\beta^{2}} \zeta^{2 n \beta}
$$

(here, as in (2), $\zeta$ is a fixed primitive $120 K$-th root of unity with $\zeta^{120}=\xi$ ). Hence

$$
\xi^{\left(n^{2}-1\right) / 120}=\frac{1}{G} \sum_{\beta \bmod 60 K} \zeta^{-\beta^{2}-1} \zeta^{2 n \beta} .
$$

Applying the above corollary to the same function $C(n)$, we see from (13) that

$$
A(\xi)=\lim _{t \searrow 0} \sum_{n=1}^{\infty} C(n) e^{-n t}=\frac{1}{G} \lim _{t \searrow 0} \sum_{\beta \bmod 60 K} \zeta^{-\beta^{2}-1} \sum_{n=1}^{\infty} \zeta^{2 n \beta} \chi_{+}(n) e^{-n t},
$$

where in the last step we have transformed $C(n)$ into a combination of terms $\zeta^{2 n \beta} \chi_{+}(n)$ using (14).

Define rational functions $\psi$ and $\phi$ by

$$
\begin{aligned}
& \psi(u)=\sum_{n=1}^{\infty} \chi_{+}(n) u^{n}=\frac{u+u^{11}+u^{19}+u^{29}}{1+u^{30}}=\frac{\left(u^{5}+u^{-5}\right)\left(u^{9}+u^{-9}\right)}{u^{15}+u^{-15}} \\
& \phi(u)=-\sum_{\substack{n \geq-1 \\
n \neq 1}} \chi_{+}(n) u^{n}=u+u^{-1}-\psi(u)=\frac{\left(u^{6}-u^{-6}\right)\left(u^{10}-u^{-10}\right)}{u^{15}+u^{-15}}
\end{aligned}
$$

where the equalities follow by summing geometric progressions. Then

$$
A(\xi)=\frac{1}{G} \lim _{t \searrow 0} \sum_{\beta \bmod 60 K} \zeta^{-\beta^{2}-1} \psi\left(\zeta^{2 \beta} e^{-t}\right) .
$$

Changing from $\psi$ to $\phi$ introduces a term

$$
\frac{1}{G} \sum_{\beta \bmod 60 K} \zeta^{-\beta^{2}-1}\left(\zeta^{2 \beta}+\zeta^{-2 \beta}\right)=2,
$$

so

$$
1-\frac{1}{2} A(\xi)=\frac{1}{2 G} \lim _{t \searrow 0} \sum_{\beta} \zeta_{(\bmod 60 K)} \zeta^{\beta^{2}-1} \phi\left(\zeta^{2 \beta} e^{-t}\right) .
$$

The terms with $K \nmid \beta$ give precisely the expression for $W(\xi)$ in (2), so to complete the proof of Theorem 1 we need only show that the terms with $K \mid \beta$ sum to 0 . This can be done easily using various symmetries, e.g. the terms with $\beta=K m$ with $m$ odd cancel in pairs under the involution $m \mapsto 30-m$, and similarly for the other terms.

Proof of Theorem 2. From the proposition, applied to $C(n)=\chi_{+}(n)$, we find that the asymptotic expansion of $A(q)$ near $q=1$ is

$$
e^{-t / 120} A\left(e^{-t}\right)=\sum_{n=1}^{\infty} \chi_{+}(n) e^{-n^{2} t / 120} \sim \sum_{r \geq 0} \frac{L\left(-2 r, \chi_{+}\right)}{r !}\left(-\frac{t}{120}\right)^{r}
$$


where $L\left(s, \chi_{+}\right)=\sum_{n=1}^{\infty} \chi_{+}(n) n^{-s}$ and we have explicitly

$$
L\left(-2 r, \chi_{+}\right)=-\frac{60^{2 r}}{2 r+1} \sum_{n=1}^{60} \chi_{+}(n) B_{2 r+1}\left(\frac{n}{60}\right) .
$$

Thus $e^{-t / 120}\left(2-A\left(e^{-t}\right)\right) \sim \sum_{r=0}^{\infty} c_{r}(-30 t)^{r} / r$ ! where

$$
c_{r}=\frac{2}{60^{2 r}}+\frac{1}{2 r+1} \sum_{n=1}^{60} \chi_{+}(n) B_{2 r+1}\left(\frac{n}{60}\right)=\left(\bar{B}-\frac{4}{15}\right)^{2 r}-\left(\bar{B}-\frac{1}{15}\right)^{2 r} .
$$

The last equality uses $\bar{B}_{m}=2\left[B_{m+1}(3 / 4)-B_{m+1}(1 / 4)\right] /(m+1)$, the relation between the $\bar{B}$ numbers and Bernoulli numbers, along with symmetries of the Bernoulli polynomials. The theorem now follows on comparison with (6).

Thus the series defined through complex asymptotics in a radial limit is identical to that emerging using congruence properties of the coefficients in the polynomial expressions arising from values at prime order roots of unity $\zeta_{K}$ as $K$ increases. We have also established the formulas given at the end of $\S 2$ : the coefficient $a_{r}$ in (9) is equal to $\frac{1}{2}(-1)^{r} L\left(-2 r, \chi_{+}\right)$, the trigonometric function appearing in equation (10) is $\frac{1}{2} \psi\left(e^{-i x}\right)$, and the relationship expressed by formulas (9) and (10) is simply the special case $C(n)=\chi_{+}(n)$ of the Proposition above.

4. Modular properties. Define a Dirichlet character $\varepsilon$ of character 5 (square root of the Legendre symbol modulo 5) by

$$
\begin{array}{l|rrrrr}
n(\bmod 5) & 0 & 1 & 2 & 3 & 4 \\
\hline \varepsilon(n) & 0 & 1 & -i & i & -1
\end{array}
$$

and denote by $\varepsilon_{+}(n)$ and $\varepsilon_{-}(n)$ the real and imaginary parts of $\varepsilon(n)$, respectively. We denote by $\chi$ the primitive character of conductor 60 defined by $\chi(n)=\left(\frac{12}{n}\right) \varepsilon(n)$ and by $\chi_{ \pm}(n)=\left(\frac{12}{n}\right) \varepsilon_{ \pm}(n)$ its real and imaginary parts, given explicitly by the formulae

$$
\begin{aligned}
& \chi_{+}(n)= \begin{cases}(-1)^{[n / 30]} & \text { if } n^{2} \equiv 1(\bmod 120), \\
0 & \text { otherwise }\end{cases} \\
& \chi_{-}(n)= \begin{cases}(-1)^{[n / 30]} & \text { if } n^{2} \equiv 49(\bmod 120) \\
0 & \text { otherwise }\end{cases}
\end{aligned}
$$

(The function $\chi_{+}$, of course, is the same one as was used in $\S 2$ and $\S 3$.) The 2dimensional space spanned by the theta functions

$$
\Theta_{\chi}(z)=\frac{1}{2} \sum_{n \in \mathbb{Z}} n \chi(n) q^{n^{2} / 120}, \quad \Theta_{\bar{\chi}}(z)=\frac{1}{2} \sum_{n \in \mathbb{Z}} n \bar{\chi}(n) q^{n^{2} / 120} \quad\left(z \in \mathfrak{H}, q=e^{2 \pi i z}\right)
$$

of weight $3 / 2$ is also spanned by the two functions

$$
\begin{aligned}
& \Theta_{+}(z)=\frac{1}{2} \sum_{n \in \mathbb{Z}} n \chi_{+}(n) q^{n^{2} / 120}=q^{1 / 120}\left(1+11 q+19 q^{3}+29 q^{7}-31 q^{8}-41 q^{14}-\cdots\right), \\
& \Theta_{-}(z)=\frac{1}{2} \sum_{n \in \mathbb{Z}} n \chi_{-}(n) q^{n^{2} / 120}=q^{49 / 120}\left(7+13 q+17 q^{2}+23 q^{4}-37 q^{11}-43 q^{15}-\cdots\right) .
\end{aligned}
$$


This space is invariant (in weight $3 / 2$ ) under the full modular group $S L(2, \mathbb{Z})$. Indeed, the obvious identities

$$
\Theta_{+}(z+1)=\zeta \Theta_{+}(z), \quad \Theta_{-}(z+1)=\zeta^{49} \Theta_{-}(z) \quad\left(\zeta=e^{\pi i / 60}\right)
$$

together with the relations $\Theta_{+}=\frac{1}{2}\left(\Theta_{\chi}+\Theta_{\bar{\chi}}\right), \Theta_{-}=\frac{1}{2 i}\left(\Theta_{\chi}-\Theta_{\bar{\chi}}\right)$ give the transformation formula

$$
\left(\begin{array}{c}
\Theta_{\chi}(z+1) \\
\Theta_{\bar{\chi}}(z+1)
\end{array}\right)=\zeta^{25}\left(\begin{array}{cc}
\cos \frac{2 \pi}{5} & -i \sin \frac{2 \pi}{5} \\
-i \sin \frac{2 \pi}{5} & \cos \frac{2 \pi}{5}
\end{array}\right)\left(\begin{array}{c}
\Theta_{\chi}(z) \\
\Theta_{\chi}(z)
\end{array}\right)
$$

with respect to the generator $\left(\begin{array}{ll}1 & 1 \\ 0 & 1\end{array}\right)$ of $S L(2, \mathbb{Z})$, while the transformation behaviour under the second generator $\left(\begin{array}{cc}0 & -1 \\ 1 & 0\end{array}\right)$ is given by the Poisson summation formula as

$$
\left(\begin{array}{c}
\Theta_{\chi}(-1 / z) \\
\Theta_{\bar{\chi}}(-1 / z)
\end{array}\right)=\left(\frac{z}{i}\right)^{3 / 2}\left(\begin{array}{cc}
0 & G_{\chi} / i \sqrt{60} \\
G_{\bar{\chi}} / i \sqrt{60} & 0
\end{array}\right)\left(\begin{array}{c}
\Theta_{\chi}(z) \\
\Theta_{\bar{\chi}}(z)
\end{array}\right)
$$

where

$$
G_{\chi}=\sum_{n(\bmod 60)} \chi(n) e^{\pi i n / 30}=-\sqrt{30+6 \sqrt{5}}+i \sqrt{30-6 \sqrt{5}}, \quad G_{\bar{\chi}}=-\bar{G}_{\chi}
$$

are the Gauss sums associated to $\chi$ and $\bar{\chi}$. In terms of the basis $\Theta_{+}$and $\Theta_{-}$, the transformation properties become

$$
\begin{aligned}
& \left(\begin{array}{l}
\Theta_{+}(z+1) \\
\Theta_{-}(z+1)
\end{array}\right)=\left(\begin{array}{cc}
\zeta & 0 \\
0 & \zeta^{49}
\end{array}\right)\left(\begin{array}{c}
\Theta_{+}(z) \\
\Theta_{-}(z)
\end{array}\right) \\
& \left(\begin{array}{l}
\Theta_{+}(-1 / z) \\
\Theta_{-}(-1 / z)
\end{array}\right)=\left(\frac{z}{i}\right)^{3 / 2}\left(\begin{array}{cc}
a & b \\
b & -a
\end{array}\right)\left(\begin{array}{l}
\Theta_{+}(z) \\
\Theta_{-}(z)
\end{array}\right)
\end{aligned}
$$

where $a=\sqrt{\frac{1}{2}\left(1-\frac{1}{\sqrt{5}}\right)}$ and $b=\sqrt{\frac{1}{2}\left(1+\frac{1}{\sqrt{5}}\right)}$.

We now introduce the Eichler integrals and period functions associated to these theta-series. Recall that in the classical theory of modular forms one associates to a cusp form $f(z)=\sum_{n=1}^{\infty} a(n) q^{n}$ of integral weight $k \geq 2$ its "Eichler integral" $\tilde{f}(z)=$ $\sum_{n=1}^{\infty} n^{-k+1} a(n) q^{n}=(k-1)$-fold primitive of $f$. The function $\tilde{f}$ is not modular, but is "nearly modular" of weight $2-k$ : the difference between $(c z+d)^{k-2} \widetilde{f}\left(\frac{a z+b}{c z+d}\right)$ and $\tilde{f}(z)$ is a polynomial. More precisely, this difference equals $r_{-d / c}(z)$, where $r_{\alpha}(z)$ for any $\alpha \in \mathbb{Q}$ is (up to a simple scalar factor) defined as $\int_{\alpha}^{\infty}(\tau-z)^{k-2} f(\tau) d \tau$.

For non-integral weights $k$, the interpretation of $\tilde{f}$ as a $(k-1)$-fold primitive no longer applies, but the definition as a sum still makes sense. We define

$$
\widetilde{\Theta}_{ \pm}(z)=\sum_{n=1}^{\infty} \chi_{ \pm}(n) q^{n^{2} / 120} \quad(z \in \mathfrak{H}) .
$$

In particular, $\widetilde{\Theta}_{+}(z)$ is $q^{1 / 120}$ times the function $A(q)$ defined in (11). (Notice that, because the theta-series $\Theta_{ \pm}$has only exponents which are a fixed multiple of perfect squares, the factor $n^{1-k}=n^{-1 / 2}$ in the definition of $\tilde{f}$ does not introduce any irrationalities, as it would for a more general function of half-integral weight, and that the formula for $\widetilde{\Theta}_{ \pm}$is actually simpler than that for $\Theta_{ \pm}$itself.) Similarly, the function 
$r_{f}$ can no longer be a polynomial, since its expected degree, $k-2$, is no longer a non-negative integer, but we can still define it as an integral. We set

$$
r_{\alpha}^{ \pm}(z)=\sqrt{\frac{2 i}{15}} \int_{\alpha}^{\infty} \frac{\Theta_{ \pm}(\tau) d \tau}{\sqrt{\tau-z}} \quad\left(\alpha \in \mathbb{Q}, \quad z \in \mathfrak{H}^{-}\right) .
$$

This function is originally defined and holomorphic in the complex lower half-plane $\mathfrak{H}^{-}=\{z \in \mathbb{C} \mid \Im(z)<0\}$, but clearly extends as a $C^{\infty}$ function to $\partial \mathfrak{H}^{-}=\mathbb{R}$ and is real-analytic on $\mathbb{R} \backslash\{\alpha\}$. Indeed, by choosing the path of integration in (16) to be an arbitrary path $L$ from $\alpha$ to $i \infty$ in $\mathfrak{H}$, we obtain a holomorphic continuation of $r_{\alpha}^{ \pm}(z)$ from $\mathfrak{H}^{-}$to $\mathbb{C} \backslash L$.

The relationship between $\widetilde{\Theta}_{ \pm}(z)$ and $r_{a}^{ \pm}(z)$, however, is not as straightforward as in the case of integral weight, and indeed, the function $\widetilde{\Theta}_{ \pm}(z)$ has no modular properties in the upper half-plane. However, its limiting values at rational points, and more generally the asymptotic expansions of $\widetilde{\Theta}_{ \pm}(\alpha+i y)$ in powers of $y$ as $y \searrow 0$ for rational numbers $\alpha$, do have modular properties. We explain this only briefly, leaving a more detailed exposition to the later paper [18]. The key idea is to introduce a second analogue of the classical Eichler integral, this time non-holomorphic and defined in the lower rather than the upper half-plane:

$$
\Theta_{ \pm}^{*}(z)=\sqrt{\frac{2 i}{15}} \int_{\bar{z}}^{\infty} \frac{\Theta_{ \pm}(\tau) d \tau}{\sqrt{\tau-z}} \quad\left(z \in \mathfrak{H}^{-}\right)
$$

Then one has the following properties:

(i) The functions $\widetilde{\Theta}_{ \pm}$and $\Theta_{ \pm}^{*}$ agree to infinite order at rational points.

(ii) The function $\Theta_{ \pm}^{*}(z)$ is nearly modular of weight $1 / 2$ in the lower half-plane, the discrepancy being given exactly by the cocycle $r_{a}^{ \pm}(z)$.

More precisely, (i) means that the asymptotic expansions of $\widetilde{\Theta}_{ \pm}(\alpha+i y)(y>0)$ and $\Theta_{ \pm}^{*}(\alpha+i y)(y<0)$ as power series in $y$ coincide for all rational numbers $\alpha$, while (ii) means that if the modular transformation properties of the weight $3 / 2$ theta-series $\Theta_{ \pm}(z)$ are given by

$$
\left(\frac{c z+d}{i}\right)^{-3 / 2}\left(\begin{array}{c}
\Theta_{+}(\gamma(z)) \\
\Theta_{-}(\gamma(z))
\end{array}\right)=M_{\gamma}\left(\begin{array}{c}
\Theta_{+}(z) \\
\Theta_{-}(z)
\end{array}\right) \quad\left(\gamma=\left(\begin{array}{ll}
a & b \\
c & d
\end{array}\right) \in S L(2, \mathbb{Z}), c>0, z \in \mathfrak{H}\right)
$$

for some matrix $M_{\gamma} \in G L(2, \mathbb{C})$ (cf. (15)), then the functions $\Theta_{ \pm}^{*}(z)$ transform by

$$
\left(\frac{c z+d}{-i}\right)^{-1 / 2}\left(\begin{array}{c}
\Theta_{+}^{*}(\gamma(z)) \\
\Theta_{-}^{*}(\gamma(z))
\end{array}\right)+M_{\gamma}\left(\begin{array}{c}
\Theta_{+}^{*}(z) \\
\Theta_{-}^{*}(z)
\end{array}\right) \doteq M_{\gamma}\left(\begin{array}{c}
r_{-d / c}^{+}(z) \\
r_{-d / c}^{-}(z)
\end{array}\right) \quad\left(z \in \mathfrak{H}^{-}\right) .
$$

We sketch the proofs briefly. For (i) we substitute the series expansion of $\Theta_{ \pm}(\tau)$ into the integral defining $\Theta_{ \pm}^{*}(z)$ and integrate term by term to obtain

$$
\Theta_{ \pm}^{*}(z)=\sum_{n=1}^{\infty} \chi_{ \pm}(n) \operatorname{erfc}(\pi \sqrt{n y / 30}) q^{-n^{2} / 120} \quad\left(z=x+i y \in \mathfrak{H}^{-}, q=e^{2 \pi i z}\right)
$$

where $\operatorname{erfc}(x)=\frac{2}{\sqrt{\pi}} \int_{x}^{\infty} e^{-u^{2}} d u$ is the complementary error function. The series converges absolutely because $\operatorname{erfc}(x)=\mathrm{O}\left(x^{-1} e^{-x^{2}}\right)$ for $x \rightarrow \infty$ and formally gives $\Theta_{ \pm}^{*}(\xi)=\sum \chi_{ \pm}(n) \xi^{n^{2} / 120}=\widetilde{\Theta}_{ \pm}(\xi)$ as $q \rightarrow \xi$ because erfc $(0)=1$. To make this rigorous we argue along the same lines as in the proof of Theorem 1, by computing the Mellin transform $\int_{0}^{\infty} \Theta_{ \pm}^{*}(\alpha+i y) y^{s-1} d y$. This Mellin transform is the product of 
two factors. One is the $L$-series $\sum_{n=1}^{\infty} \chi_{ \pm}(n) \xi^{n^{2} / 120} n^{-2 s}$, whose values at negative half-integers and integers we already know to be equal to 0 and to the appropriately normalised Taylor coefficients of $\widetilde{\Theta}_{ \pm}(\alpha+i y)$, respectively. The other is the Mellin transform of $e^{\pi y / 60} \operatorname{erfc}(\sqrt{y / 30})$, which is the sum of the power series $e^{\pi y / 60}$ and an odd power series in $\sqrt{y}$ (because $\operatorname{erfc}(x)$ is the sum of 1 and an odd power series in $x$ ), and hence has simple poles at negative integers and half-integers, with the values at the former being the same as for the Mellin transform of $e^{-\pi y / 60}$, but with alternating signs. The assertion about the asymptotic expansions of $\widetilde{\Theta}_{ \pm}$and $\Theta_{ \pm}^{*}$ follows. Assertion (ii) follows in a more straightforward way by replacing $\tau$ by $\gamma(\tau)$ in the integral defining $\Theta_{ \pm}^{*}(\gamma(z))$ and making the evident change of variables to transform it into an integral from $\bar{z}$ to $\gamma^{-1}(\infty)=-d / c$ and then combining this with the integral defining $\Theta_{ \pm}^{*}(z)$ to get an integral going from $-d / c$ to $\infty$.

Combining (i), (ii) and the assertion about the smoothness (indeed, almost-everywhere analyticity) of the function $r_{\alpha}^{ \pm}(z)$ on the real line, we find that the asymptotic expansions of our "Eichler integrals" $\widetilde{\Theta}_{ \pm}(z)$ near rational points have a modular property modulo smooth functions. In particular, if we ignore the full asymptotic expansions and concentrate on just the values at rational points, then we see that the values $\widetilde{\Theta}_{ \pm}(\alpha)$ at rational points $\alpha$ (defined, as usual, by the limits from above) are modular of weight $1 / 2$ modulo smooth functions of $\alpha$. In particular, for $\alpha=0$ and our original function $A(q)=q^{-1 / 120} \Theta_{+}^{*}(z)$ we deduce for the values $W\left(e^{2 \pi i / K}\right)=1-\frac{1}{2} A\left(e^{2 \pi i / K}\right)$ the asymptotic expansion

$$
W\left(e^{2 \pi i / K}\right) \sim \sqrt{K / i} e^{-\pi i / 60 K}\left(a e^{-\pi i K / 60}+b e^{-49 \pi i K / 60}\right)+W_{\infty}\left(e^{2 \pi i / K}-1\right)
$$

as $K \rightarrow \infty$, with $a$ and $b$ as in equation (15). This agrees with and explains the asymptotic properties coming from the stationary phase description (PE) of the original Witten integral (the three terms in (18) correspond precisely to the contributions from the three equivalence classes of flat connections on $\Sigma(2,3,5)$, as described in equations (7) and (8)), but now in a more general context, since we can give such expansions around any rational point and can also see that the value of the leading term is determined by a modular transformation property under the full modular group.

5. Relation to Ramanujan's mock theta functions. In a singularly prescient definition, Ramanujan, in his famous last letter to Hardy in January 1920, introduced the terminology "mock theta function" to describe certain power series in $q=e^{2 \pi i z}$ which, though not modular, possess asymptotic expansions for $q$ tending to roots of unity of the sort normally associated with modular functions and forms. His definition and examples were highly enigmatic (as witness the title of Watson's famous talk on the subject, "The Final Problem"), and while the various identities which he wrote down in this letter and in his "Lost Notebook" have now all been proved, the subject retains an aura of mystery. The same phenomenon of "interesting asymptotic behaviour near roots of unity" (or, if one will, of the "quantisation of modular forms") is of course the theme of this paper, so it comes as a pleasant surprise that Ramanujan's mock theta functions are not only similar in spirit, but in fact directly related, to our function $W(\xi)$. This observation is due to Sander Zwegers (Utrecht University) and is very recent; we thank him for permitting us to quote some of his results here.

Ramanujan's letter speaks of mock theta functions of the third, fifth, and seventh order (terminology not defined). We are concerned with those of fifth order. We recall some of his statements. (A good reference is [1], particularly Sections 1 and 7.) In his 
letter, Ramanujan defines (among others) three power series, all defined for $|q|<1$,

$$
\begin{aligned}
& f(q)=\sum_{n=0}^{\infty} \frac{q^{n^{2}}}{(1+q)\left(1+q^{2}\right) \cdots\left(1+q^{n}\right)} \\
& \phi(q)=\sum_{n=0}^{\infty} q^{n^{2}}(1+q)\left(1+q^{3}\right) \cdots\left(1+q^{2 n-1}\right) \\
& \psi(q)=\sum_{n=1}^{\infty} q^{n(n+1) / 2}(1+q)\left(1+q^{2}\right) \cdots\left(1+q^{n-1}\right)
\end{aligned}
$$

and states without proof the relations

$$
\phi\left(-q^{2}\right)+\psi(-q)=2 \phi\left(-q^{2}\right)-f(q)=\theta_{1}(q) P(q),
$$

where $\theta_{1}(q)$ and $P(q)$ are the standard modular forms (of weights $\frac{1}{2}$ and 0 , respectively) defined by

$$
\theta_{1}(q)=\sum_{n \in \mathbb{Z}}(-1)^{n} q^{n^{2}}=\prod_{n>0} \frac{1-q^{n}}{1+q^{n}}, \quad P(q)=\prod_{\substack{n>0 \\ n \equiv \pm 1(5)}} \frac{1}{1-q^{n}}=\frac{\sum_{n}(-1)^{n} q^{\left(5 n^{2}-n\right) / 2}}{\prod_{n>0}\left(1-q^{n}\right)}
$$

Furthermore, in his "Lost Notebook" he gives the futher identity

$$
f(q)=\theta_{1}\left(q^{5}\right) P(q)-2 \Phi\left(q^{2}\right),
$$

where the power series $\Phi(q)$ is defined by

$$
\Phi(q)=-1+\sum_{n=0}^{\infty} \frac{q^{5 n^{2}}}{(1-q)\left(1-q^{4}\right) \cdots\left(1-q^{5 n-1}\right)\left(1-q^{5 n+1}\right)} .
$$

These formulas resisted proof for many years and were finally settled by Hickerson [5] in 1988.

Now the series defining $\Phi(q)$ converges not only inside, but also outside (though not on!) the unit circle, the function $\Phi^{*}(q)=\Phi(1 / q)$ being given for $|q|<1$ by

$$
\Phi^{*}(q)=-1-\sum_{n=0}^{\infty} \frac{q^{5 n+1}}{(1-q)\left(1-q^{4}\right) \cdots\left(1-q^{5 n-1}\right)\left(1-q^{5 n+1}\right)} .
$$

For the power series expansion of this new function Zwegers proves the identities

$$
-\Phi^{*}(q)=A_{+}(q)-P(q) F_{+}(q)=A_{-}(q)+P(q) F_{-}(q),
$$

where $A_{ \pm}$and $F_{ \pm}$are defined by

$$
A_{ \pm}(q)=\sum_{\substack{n>0 \\ n \equiv \pm 1(5)}}\left(\frac{12}{n}\right) q^{\left(n^{2}-1\right) / 120}, \quad F_{ \pm}(q)=\sum_{ \pm\left(n-\frac{1}{2}\right)>0}(-1)^{n} q^{\left(5 n^{2}-n\right) / 2} .
$$

The sum of the functions $A_{+}$and $A_{-}$is precisely the function $A(q)$ defined by (11), and the three functions $A_{+}, A_{-}$and $\frac{1}{2} A(q)$ have the same asymptotic behaviour at all roots of unity. The function $f(q)$ has a finite value at all roots of unity of odd order, and we can use the above identities to relate the expansions of $A(q)$ to that of the functions $\Phi, f, \phi$ and $\psi$ at the appropriate roots of unity. In particular, from 
Zwegers's results we deduce the beautiful formula

$$
W(\xi)=1+\sum_{n=1}^{\infty} x^{-n^{2}}(1+x)\left(1+x^{2}\right) \cdots\left(1+x^{n-1}\right)
$$

for the WRT-invariant of the Poincaré sphere, where $\xi$ as usual is a $K$ th root of unity and $x$ is a square root of $\xi$ of exact order $2 K$. The sum on the right is written as an infinite one, but in fact terminates after $K$ terms, since $1+x^{K}$ vanishes. More generally, if we replace $\xi$ by $\xi e^{-t}$ and $x$ by $x e^{-t / 2}$ in (20), then the right-hand side makes sense as a power series in $t$, since the $n$th term vanishes to order at least $M$ for $n \geq(2 M-1) K$, and gives the correct asymptotic expansion of $W\left(\xi e^{-t}\right)$. This is to be compared with the similar behaviour of the function studied in [17], which is also related to the Eichler integral of a theta series, but this time of weight $1 / 2$ rather than $3 / 2$.

Finally, we mention another and even simpler formula for $A(q)$, also pointed by Zwegers, which can be obtained by a simple substitution from equation (23) of [17]:

$$
A_{+}(q)=\sum_{n=0}^{\infty} q^{n}(1-q)\left(1-q^{6}\right) \cdots\left(1-q^{5 n-4}\right) .
$$

There is, of course, a similar formula for $A_{-}$as well. These formulas can be used to compute the value of $A(q)$ at (and its asymptotic expansion near) all roots of unity of order not divisible by 5 .

6. Generalisation to other manifolds. Consider an arbitrary Seifert fibred manifold, $M=\Sigma\left(\frac{P_{1}}{Q_{1}}, \ldots, \frac{P_{N}}{Q_{N}}\right)$ fibred over $S^{2}$. According to [10], the WRT-invariant $Z_{K}(M)$ equals $W_{M}\left(\zeta_{K}\right) /\left(\zeta_{K}-1\right) \zeta_{K}^{\sigma}$, where $\sigma$ is a certain rational number defined in terms of Dedekind sums $(\sigma=\phi / 4-1 / 2$ in the notation of [10]; $\sigma=121 / 120$ for $M=\Sigma(2,3,5))$ and $W_{M}\left(\zeta_{K}\right)$ is given by a formula analogous to $(3)$ :

$$
W_{M}\left(\zeta_{K}\right)=\frac{\operatorname{sgn} H-i}{\sqrt{P K}} \sum_{\substack{\beta(\bmod 2 P K) \\ \beta \neq 0(\bmod K)}} \frac{\prod_{j=1}^{N} \sin \left(\pi \beta / K P_{j}\right)}{(\sin (\pi \beta / K))^{N-2}} e^{-\pi i H \beta^{2} / 2 K P} .
$$

Here $P=\prod_{j} P_{j}$ and $H=P \sum_{j} \frac{Q_{j}}{P_{j}}$, so that $\left|H_{1}(M, \mathbb{Z})\right|=|H|$. Again one can replace this by a formula similar to $(2)$ which defines an invariant $W_{M}(\xi) \in \mathbb{Q}(\xi)$ for every root of unity $\xi$.

Define a simple hyperbolic function $\phi$ and an integer valued function $\chi(n)$ by

$$
\phi(x)=4 \frac{\prod_{j=1}^{N} \sinh \frac{x}{2 P_{j}}}{(\sinh x / 2)^{N-2}}=\sum_{n=1}^{\infty} \chi(n) e^{-n x / 2 P} .
$$

(The case dealt with in the rest of this paper is anomalous since $\frac{1}{2}+\frac{1}{3}+\frac{1}{5}>1$. In all other cases, the above definition of $\chi(n)$ makes sense -in that case alone, it is necessary to alter the limits to include $n=-1$ but exclude $n=1$. We now assume that this anomalous situation does not arise.) When $N=3$, so that we have three fibres, $\chi$ will be a periodic function of period $2 P$; when $N>3, \chi(n)$ will be described by polynomials in $n$ of degree $N-3$, which polynomial to use being dependent on the value of $n$ modulo $2 P$. 
Define a holomorphic function on the disc $|q|<1$ by

$$
A(q)=\sum_{n=1}^{\infty} \chi(n) q^{n^{2} / 4 P H}
$$

The same reasoning as in $\S 3$ will give the following.

THEOREM 3. For three-fibred Seifert integer homology sphere $M$ and any root of unity $\xi$, the radial limit of $-\frac{\operatorname{sgn}(H)}{2} A(q)$ as $q \rightarrow \xi$ equals $W_{M}(\xi)$.

The function $A(q)$ is the Eichler integral of a theta series of weight $\frac{3}{2}$ (defined by the same sum as for $A(q)$ but with a factor $n$ included), and we could again use the methods of $\S 4$ to obtain information about the transformation behaviour of $A\left(e^{2 \pi i \alpha}\right)$ $(\alpha \in \mathbb{Q})$ under the action of the modular group. In the general case of $N>3$ fibres we expect a similar story, but now also involving derivatives of theta functions.

\section{REFERENCES}

[1] G. E. Andrews, Mock theta functions, in Theta functions-Bowdoin 1987, Part 2, Proc. Sympos. Pure Math 49, AMS, Providence, R.I., 1989, pp. 283-298.

[2] M. F. AтזYAh, Topological quantum field theory, Publ. Math. IHES, 68 (1989), pp. 175-186.

[3] M. F. Аттун, The geometry and physics of knots, Cambridge University Press, Cambridge, 1990.

[4] D. S. FreEd AND R. E. GoMPF, Computer calculations of Witten's 3-manifold invariant, Commun. Math. Phys., 141 (1991), pp. 79-117.

[5] D. R. Hickerson, A proof of the mock theta conjectures, Invent. Math., 94 (1988), pp. 639-660.

[6] L. C. JEFFREY, Chern-Simons-Witten invariants of lens spaces and torus bundles and the semiclassical approximation, Commun. Math. Phys., 147 (1992), pp. 563-604.

[7] R. LAWrence, Asymptotic expansions of Witten-Reshetikhin-Turaev invariants for some simple 3-manifolds, J. Math. Phys., 36 (1995), pp. 6106-6129.

[8] R. LAWRENCE, Witten-Reshetikhin-Turaev invariants of 3-manifolds as holomorphic functions, Lecture Notes in Pure and Applied Mathematics, 184 (1996), pp. 363-377.

[9] R. Lawrence, An Introduction to Topological Field Theory, in The interface of knots and physics-San Francisco 1995, Proc. Symp. Appl. Math. 51, AMS, Providence, R.I., 1996, pp. 89-128.

[9] R. Lawrence, An Introduction to Topological Field Theory, Proc. Symp. Appl. Math., 51 (1996), pp. 89-128.

[10] R. LAWREnce AND L. RozAnsky, Witten-Reshetikhin-Turaev invariants of Seifert manifolds, Commun. Math. Phys., to appear (1999).

[11] H. Murakami, Quantum SU(2)-invariants dominate Casson's SU(2)-invariant, Math. Proc. Camb. Phil. Soc., 115 (1993), pp. 253-281.

[12] T. Онтsuki, A polynomial invariant of rational homology 3-spheres, Invent. Math., 123 (1996), pp. 241-257.

[13] N. YU. REShETIKHIN AND V. TURAev, Invariants of 3-manifolds via link polynomials and quantum groups, Invent. Math., 103 (1991), pp. 547-597.

[14] L. RoZANSKY, On p-adic properties of the Witten-Reshetikhin-Turaev invariant, Preprint (1998).

[15] J. SinK, Asymptotic expansions of quantum invariants and a zeta-function of a knot, Thesis, University of Michigan (1999).

[16] E. Witten, Quantum Field Theory and the Jones Polynomial, Commun. Math. Phys., 121 (1989), pp. 351-399.

[17] D. ZAGIER, Vassiliev invariants and a strange identity related to the Dedekind eta-function, Preprint (1998).

[18] D. ZAGIER, Period functions for modular forms of half-integral weight, in preparation. 
